\title{
PLANT PHYLOGENY AS A MAJOR PREDICTOR OF FLOWER VISITATION BY NITIDULID BEETLES, A LINEAGE OF ANCESTRAL ANGIOSPERM
}

\section{POLLINATORS}

\author{
Carlos M. Herrera ${ }^{1 *}$, J. Carlos Otero ${ }^{2}$ \\ ${ }^{1}$ Estación Biológica de Doñana, Consejo Superior de Investigaciones Científicas, Americo Vespucio 26, E-41092 Sevilla, Spain \\ ${ }^{2}$ Departamento de Biología Animal, Facultad de Biología, Universidad de Santiago, E-15782 Santiago de Compostela, \\ Spain
}

Journal of Pollination Ecology, 29(14), 2021, pp 179-188

DOI: $10.26786 / 1920-$

$7603(2021) 636$

Received 24 January 2021, accepted 03 May 2021

*Corresponding author: herrera@ebd.csic.es

\begin{abstract}
Plant phylogeny sometimes predicts interspecific variation in pollinator composition better than gross floral features, and its predictive value seems to differ among major groups of insect pollinators. Pollination by beetles exhibits the strongest phylogenetic signal and the strongest phylogenetic conservatism, which is particularly intriguing given that beetles were probably the pollinators of early angiosperms. We examine in this paper the relationship between plant phylogeny and flower visitation by nitidulid beetles (Coleoptera: Nitidulidae), an old monophyletic group of flower specialists and pollinators of gymnosperms and angiosperms. Using quantitative data on pollinator composition for 251 plant species (belonging to 167 genera in 46 families) from well-preserved Mediterranean montane habitats from southeastern Spain, the following questions were addressed: Is pollination by nitidulids correlated with plant phylogeny in the large species sample studied, and if it does, which are the relative importances of plant phylogeny, floral characteristics, and environmental features as predictors of nitidulid pollination in the plant assemblage studied? Nitidulids were recorded in flowers of $25 \%$ of the plant species considered. Their distribution was significantly related to plant phylogeny, being clustered on certain lineages (Ranunculales, Malvales, Rosales, Asterales) and remarkably absent from others (e.g., Fabales, Lamiales). None of the environmental (habitat type, elevation) or macroscopic floral features considered (perianth type and color, flower mass) predicted nitidulid visitation after statistically accounting for the effect of plant phylogeny. We theorize that nitidulid beetles use characters of plants that track plant phylogeny at least as deep as the early radiation of the eudicots, imaginably characters such as the chemical signatures of pollen.
\end{abstract}

Keywords-Beetle pollination, Coleoptera, Mediterranean habitats, Nitidulidae, plant phylogeny, phylogenetic signal

\section{INTRODUCTION}

In a large, taxonomically diverse sample of insect-pollinated species with similar historical and ecological backgrounds, it was recently shown that plant phylogeny can sometimes be a better predictor of interspecific variation in pollinator composition than gross floral features as scored by the human eye (Herrera 2020). Interestingly, this study found that the magnitude of phylogenetic signal (sensu Blomberg \& Garland 2002) and extent of phylogenetic conservatism (sensu Losos 2008) of interspecific variation in pollinator composition differed widely among the four major groups of insect pollinators (Coleoptera, Diptera, Hymenoptera, Lepidoptera; Herrera 2020). In particular, pollination by beetles exhibited the strongest phylogenetic signal and phylogenetic conservatism (Herrera 2020). Since beetles are generally considered the ancestral, oldest pollinators of animal-pollinated plants (Schiestl \& Dötterl 2012; Cai et al. 2018; Bao et al. 2019), strong 
phylogenetic signal and phylogenetic conservatism of beetle pollination in extant plant communities is particularly intriguing.

Strongly phylogenetically-driven patterns of beetle pollination in extant plant species assemblages could reflect long-lasting evolutionary associations between beetles and their respective plant lineages, intrinsic limitations of beetles as a group to adapt to and exploit the full range of floral resources brought about by the floral diversification of angiosperms, or some combination of these. Data on flower visitation by beetles analyzed by Herrera (2020) could not be used to scrutinize these hypotheses, since they referred to a heterogeneous assortment of beetle families presumably differing in evolutionary age, diversification history, and relationships with plants. Using flower visitation data for an extensive, phylogenetically comprehensive sample of plant species coexisting regionally in well-preserved Mediterranean montane habitats from southeastern Spain, we examine in the present paper the relationships between plant phylogeny and flower visitation by nitidulid beetles, an old monophyletic group of flower specialists and pollinators of widely divergent gymnosperms and angiosperms (Hunt et al. 2007; Kono \& Tobe 2007; Procheş \& Johnson 2009; Cline et al. 2014; Cai et al. 2018). More specifically, we will address the following questions: (1) Is pollination by nitidulid beetles correlated with plant phylogeny in the large species sample studied? (2) If it does, which are the relative importances of plant phylogeny, floral characteristics and environmental features as predictors of nitidulid visitation in the plant assemblage studied? Our results show that nitidulid pollination occurred in a substantial proportion of the species studied, was significantly related to plant phylogeny, and statistically unrelated to gross floral characteristics and environmental features after the effect of plant phylogeny was controlled for.

\section{MATERIALS AND METHODS}

\section{FIELD METHODS}

Pollinator composition data used in this paper were collected during February-December 19972020 in the Sierra de Cazorla (Jaén Province, southeastern Spain), a region characterized by well-preserved natural habitats. Data were obtained for 251 plant species in 167 genera and 46 families (Supplementary Material Table S1), using field methods described in detail by Herrera (2020, 2021). The elemental sampling unit was the "pollinator census", consisting of a 3-min watch of a flowering patch whose total number of open flowers was also counted. Only taxa whose individuals contacted anthers or stigmas, or with discernible pollen grains on their bodies, were considered as pollinators. All pollinators visiting any flower in the focal patch during the 3-min period were identified, and total number of flowers probed by each individual was recorded. A total of 31,139 censuses were conducted for all plant species combined (sampling effort data for each plant species is shown in Supplementary Material Table S1). Assessing the number of flowers visited by pollinators was impractical in species with tiny flowers packed into dense inflorescences. In these instances the number of inflorescences available per patch and visited per census were counted rather than individual flowers, and visitation rates actually refer to inflorescences. For simplicity, single flowers and dense inflorescences will both be termed "flowers" hereafter.

Due to their small size and unapparent diagnostic features, nitidulid beetles could not be identified to species in pollinator censuses. To assess the number, identity and proportions of nitidulid species involved in flower visitation, a random sample of $N=165$ individuals were collected from the flowers of 36 plant species, preserved in ethanol and identified to species (Supplementary Material Table S1). Species identification largely followed the monograph of Audisio (1993).

\section{DATA ANALYSIS}

Each plant species was assigned to one of the following nine habitat types (number of species in parentheses): vertical rock cliffs (9); sandy or rocky dolomitic outcrops (31); banks of permanent streams or flooded/damp areas around springs (20); patches of grasslands and meadows on deep soils in relatively flat terrain (52); dwarf mountain scrub dominated by cushion plants (32); forest edges and large clearings (37); forest interior (20); tall, dense Mediterranean sclerophyllous forest and scrub (19); or sites locally disturbed by 
humans, large mammals or natural abiotic processes (31). Each plant species was also characterized by the elevation of the sampling site (range $=770-1920 \mathrm{~m}$ a. s. 1.; an average figure was used for the few species sampled at more than one site). Species were also characterized by three macroscopic floral features, namely perianth type, perianth color, and flower size. Two discrete perianth classes were recognized which corresponded to open, non-restrictive perianths versus tubular or otherwise restrictive perianths. Mean dry mass of flowers was obtained for each species by weighing samples from census localities dried until constant mass. Dry mass is used here as a surrogate for overall flower size. Floral and environmental features of species studied are shown in Supplementary Material Table S2.

All statistical analyses were carried out using $R$ ( $R$ Core Team 2020). A phylogenetic tree for the set of plant species studied was obtained using the phylo.maker function in the V.Phylomaker package (Jin \& Qian 2019) and the GBOTB megatree of Smith \& Brown (2018). Statistical significance of phylogenetic signal in nitidulid flower visitation rate, defined as "a tendency for related species to resemble each other more than they resemble species drawn at random from the tree" (Blomberg \& Garland 2002), was tested with the philoSignal function in the philosignal package (Keck et al. 2016) and Moran's I method. The latter relies on an autocorrelation approach, makes no assumptions on model of change and incorporates information on branch length (Münkemüller et al. 2012). A phylogenetic regression approach based on generalized least squares (Symonds \& Blomberg 2014) was adopted to evaluate the importance of environmental (elevation, habitat type) and floral features (perianth type, perianth color, mass) as predictors of nitidulid flower visitation rates after statistically accounting for phylogenetic correlations in the data. Phylogenetic correlations were incorporated into the model by setting the variance-covariance structure between plant species to match that expected under a Brownian motion process (Symonds \& Blomberg 2014). Computations were performed with function gls in the nlme package (Pinheiro et al. 2018) and the expected covariance structure between species was obtained using the corBrownian function of the ape package (Paradis \& Schliep 2018).
The frequencies of the different nitidulid species identified in the random sample of individuals collected from flowers were used to estimate sample completeness and true regional species richness of Nitidulidae in flowers, using the interpolation-extrapolation method in the iNEXT package (Hsieh et al. 2016). Sample completeness was assessed with Good-Turing's "sample coverage" parameter (= estimate of the proportion of the total population that is represented by those species occurring in the sample; Hsieh et al. 2016).

\section{RESULTS}

Pollinator censuses yielded nitidulid beetles in 63 plant species belonging to 46 genera and 23 families (Fig. 1 gives examples). They were generally scarce, accounting for $1.62 \%$ of total pollinator individuals $(N=36,282)$ and $0.55 \%$ of total flower visits $(N=113,776)$, all plant species combined. In the subset of plants where nitidulids were recorded, their flower visitation rates (= probability of one flower being visited per minute) were usually very low, ranging between 1.56E-05 $\mathrm{min}^{-1}$ and 9.76E-03 $\mathrm{min}^{-1}$ (interquartile range $=7.81 \mathrm{E}-05-8.36 \mathrm{E}-04 \mathrm{~min}^{-1}$; Supplementary Material Table S1).

The distribution of flower visitation rates by nitidulid beetles across the phylogenetic tree depicting the evolutionary relationships among the 251 plant species studied was conspicuously clustered (Fig. 2). Within dicots, nitidulid visitation was highest in plant species belonging to a relatively small group of lineages comprising the order Ranunculales and several members of the 'superrosid' clade, including Malvales, Brassicales and Rosales. Some smaller clusters of nitidulid visitation occurred within Monocots and the Asteroideae subfamily of Asteraceae. Nearly as remarkable as the phylogenetically clustered occurrence of nitidulids in the aforementioned plant lineages was their virtual absence from the flowers of some very species-rich lineages such as Fabales and Lamiales (Fig. 2).

The relationship between nitidulid flower visitation rates and plant phylogeny illustrated graphically in Fig. 2 was corroborated analytically by the statistically significant Moran's $I$ index for phylogenetic signal $(I=0.00729, P=0.034)$. After controlling for the phylogenetic correlation, no 


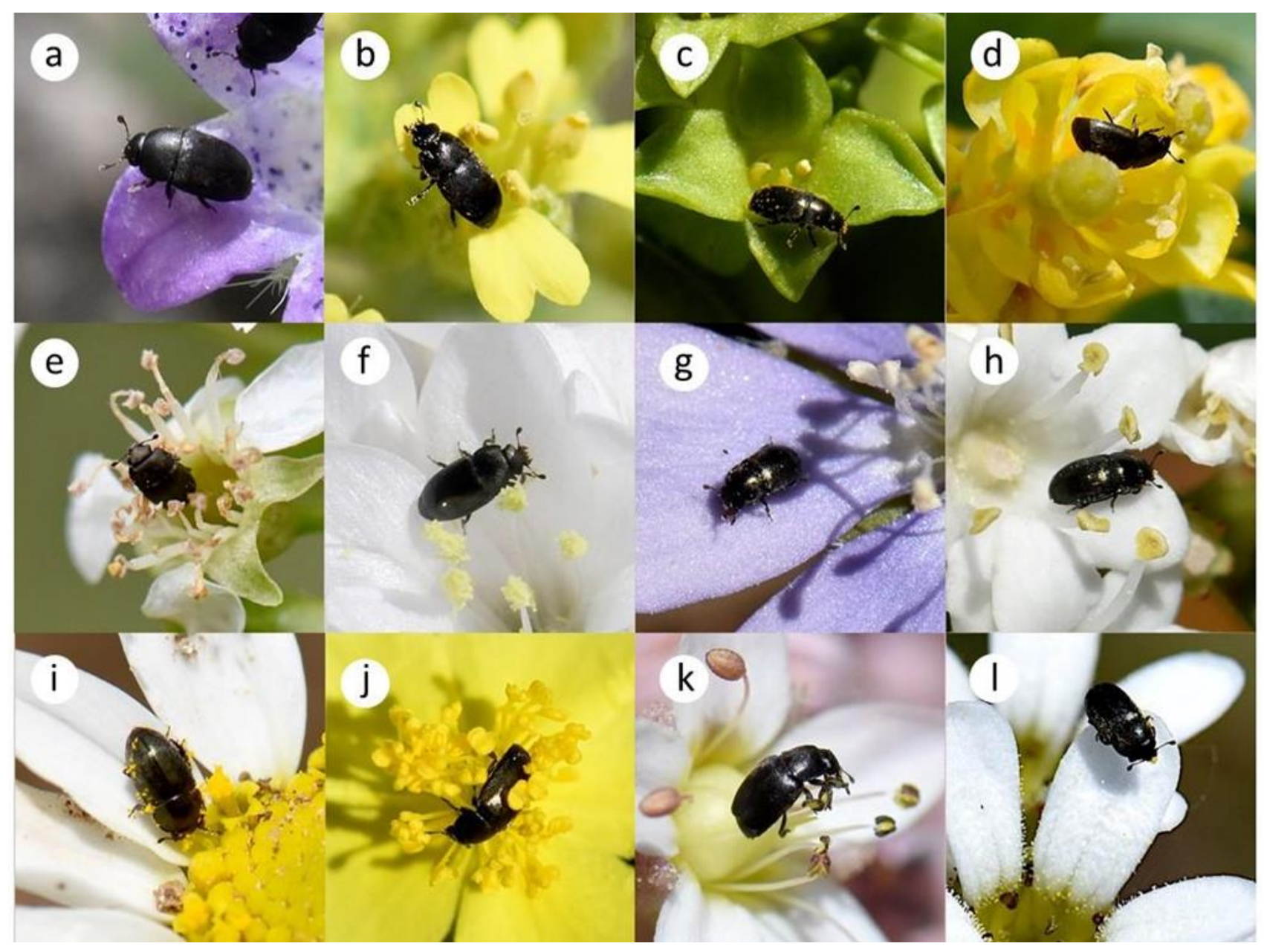

Figure 1. Nitidulid beetles in flowers, exemplifying the broad variety of flowers where they were recorded. Each picture corresponds to a different plant family: a, Campanula mollis (Campanulaceae); b, Draba hispanica (Brassicaceae); c, Daphne laureola (Thymelaeaceae); d, Berberis hispanica (Berberidaceae); e, Prunus mahaleb (Rosaceae); f, Armeria filicaulis (Plumbaginaceae); g, Hepatica nobilis (Ranunculaceae); h, Viburnum tinus (Adoxaceae); i, Anthemis pedunculata (Asteraceae); $\mathrm{j}$ Helianthemum oelandicum (Cistaceae); k, Sedum dasyphyllum (Crassulaceae); I, Saxifraga carpetana (Saxifragaceae). Although species of Nitidulidae cannot be safely identified from photographs, most individuals depicted probably are Brassicogethes aeneus.

statistically significant effects of perianth type, perianth colour, flower size, site elevation or habitat type on nitidulid flower visitation rate was found (Table 1). The effect of $\log$ flower mass approached statistical significance $(P=0.077)$, thus suggestive of a possible trend for nitidulid visitation to increase with flower size independently of phylogeny (standardized partial regression coefficient $\pm S E=0.264 \pm 0.149$ ) .

Five different species, occurring with very unequal frequencies, were identified in the random sample of nitidulid specimens collected from flowers (number of individuals in parentheses; see Supplementary Material Table S1 for distribution among plant species): Brassicogethes aeneus (124), Acanthogethes brevis (20),
Thymogethes nigritus (16), Brassicogethes fulvipes (4) and Acanthogethes lamii (1). Sample completeness estimated from these frequency data was 1.000 (95\% confidence interval $=0.999-1.001)$, and the estimated species richness of all flower-dwelling Nitidulidae in the plant community studied $( \pm$ SE) was $5.0 \pm 0.50$ species. The distribution of identified individuals of the three most frequent species on the plant phylogenetic tree is shown in Fig. 3. The distributions of Brassicogethes aeneus, the most abundant species (75\% of total), and Thymogethes nigritus largely matched the distribution for all nitidulids combined shown in Fig. 2. Individuals of Acanthogethes brevis were mostly associated with flowers of Asterales. 


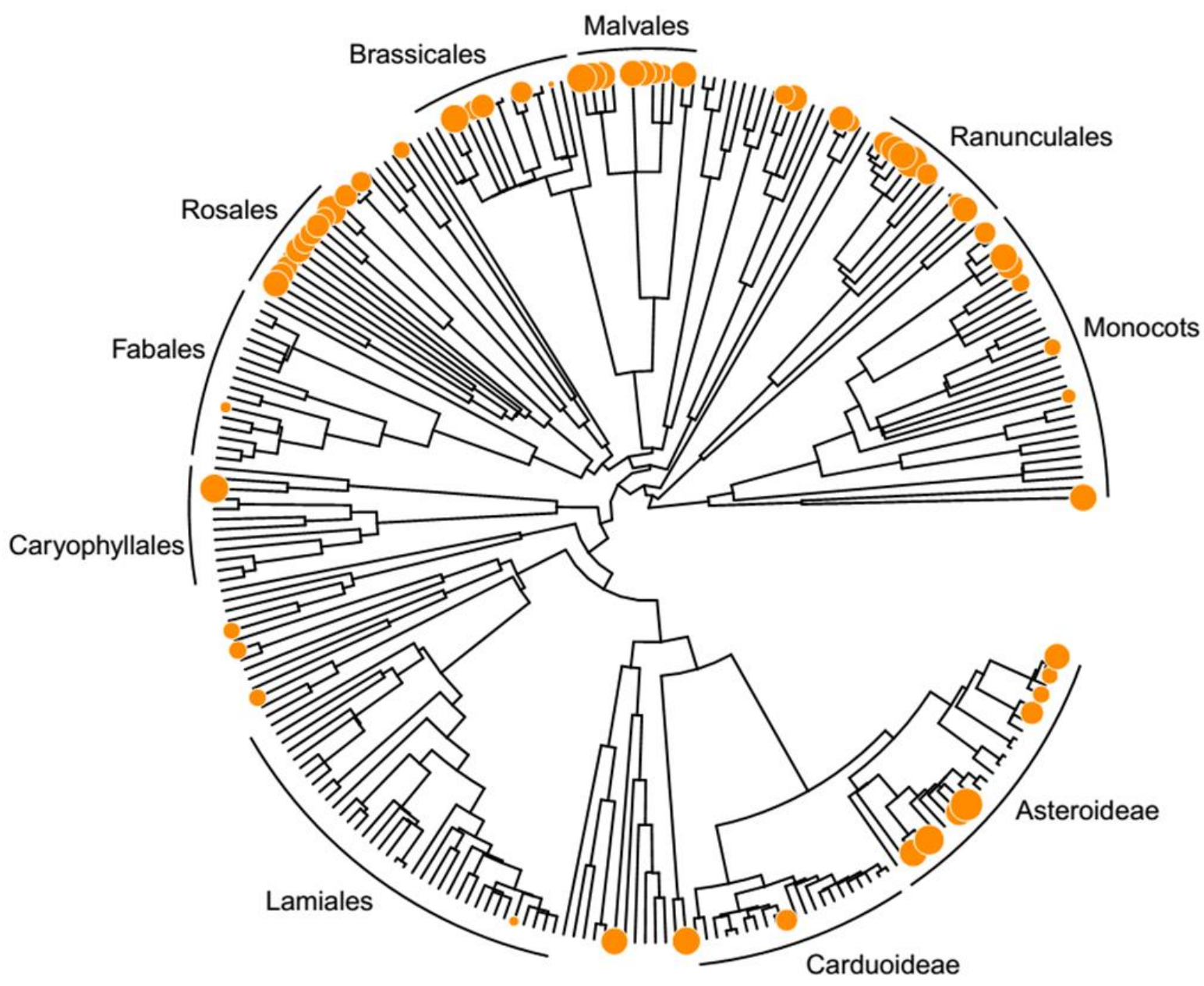

Figure 2. Distribution of flower visitation rates (symbol size) by nitidulid beetles (all species combined) across the phylogenetic tree depicting the evolutionary relationships of the 251 plant species from 46 families studied. Tree tips without symbols denote plant species where Nitidulidae were not recorded.

Table 1. Results of the phylogenetic regression across plant species $(N=251)$ testing for the effects of perianth type and color, flower mass, elevation and habitat type, on mean flower visitation probability by nitidulid beetles while simultaneously accounting for the significant phylogenetic correlation underlying flower visitation data.

\begin{tabular}{llll}
\hline Effect & $\mathrm{df}$ & $\chi 2$ & P-value \\
\hline Floral features: & & & \\
$\quad$ Perianth type & 1 & 0.057 & 0.81 \\
$\quad$ Perianth color & 7 & 1.42 & 0.98 \\
$\quad$ Flower mass (log- & 1 & 3.12 & 0.077 \\
$\quad$ transformed) & & & \\
$\quad \begin{array}{l}\text { Environmental features: } \\
\quad\end{array}$ & 1 & 0.28 & 0.59 \\
$\quad$ Elevation & & & \\
$\quad$ Habitat type & 8 & 2.06 & 0.98 \\
\hline
\end{tabular}

\section{DISCUSSION}

Many species of Nitidulidae are associated with flowers in their adult and larval stages. The adults feed on pollen and are effective pollinators of a variety of plants in tropical and non-tropical habitats worldwide (Podoler et al. 1984; Crowson 1988; Nadel \& Peña 1994; Jürgens et al. 2000; Alonso 2004; Procheş \& Johnson 2009). Although a rigorous demonstration of their pollinating effectiveness would have required controlled experiments, circumstantial evidence suggests that the Nitidulidae recorded in pollinator censuses in this study should be considered as true pollinators of most plant species where they occurred. Examination of $\sim 300$ close-up photographs of Nitidulidae taken as part of pollinator census work (Herrera 2020, 2021) revealed that they often 


\section{Acanthogethes brevis}

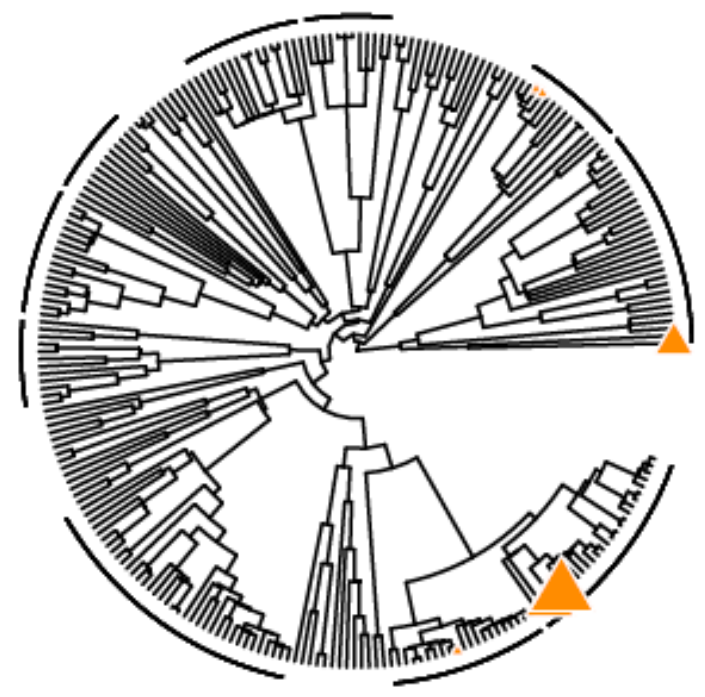

\section{Brassicogethes aeneus}

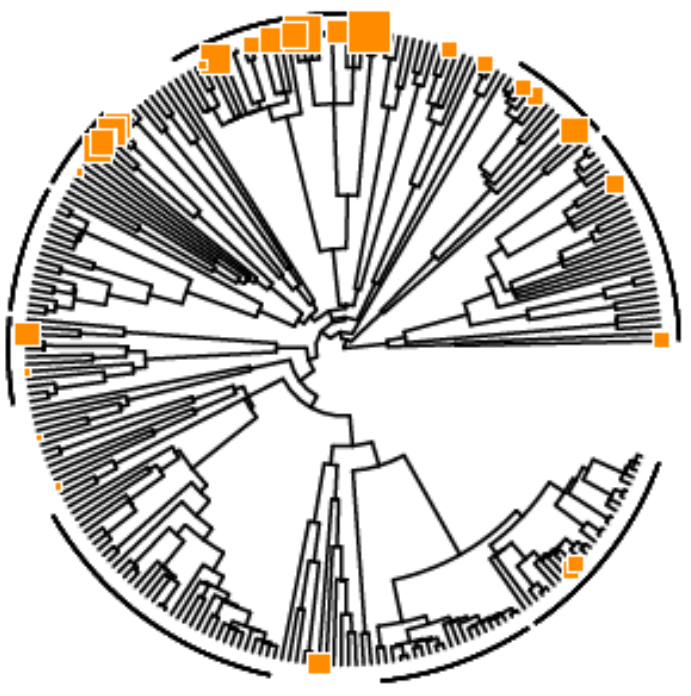

\section{Thymogethes nigritus}

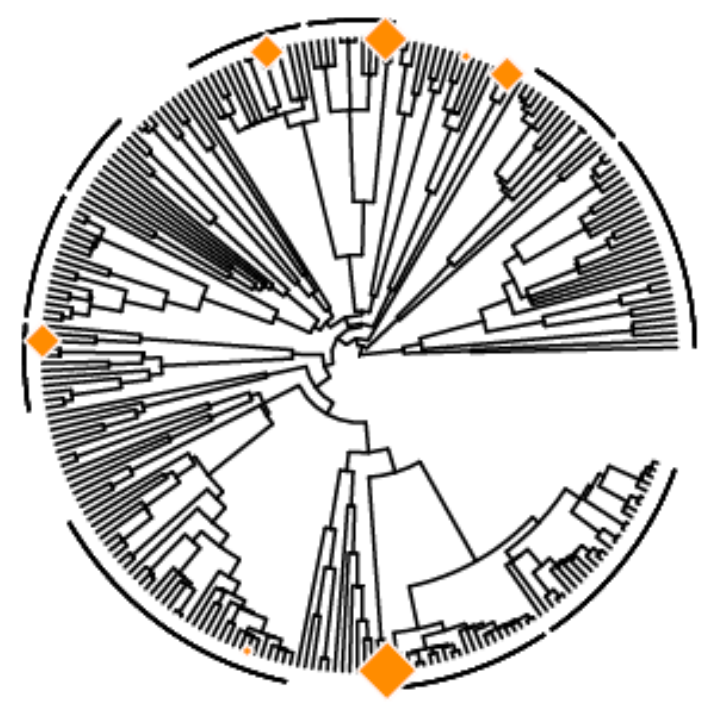

Figure 3. Distributions across the plant phylogenetic tree of the number of specimens identified (symbol size) for the three commonest species in the sample of nitidulid specimens collected (see Supplementary Material Table S1 for details). Plant clades marked by arcs match those in Figure 2.

contacted the anthers and/or stigmas of visited flowers, and that head, legs or elytra often displayed substantial numbers of visible pollen grains, as exemplified by some of the individuals depicted in Fig. 1. Due to their low flower visitation rates, however, the proportional contribution as pollinators was probably low for most plant species included in this study. An outstanding exception is Daphne laureola (Thymelaeaceae; Fig. 1c). In this plant, nitidulid beetles accounted for $85 \%$ of all pollinators recorded in censuses (Herrera 2020: Appendix S3), and experimental work done in the same region as the present investigation demonstrated that they were important pollinators (Alonso 2004).

In the Mediterranean montane habitats studied, nitidulid beetles were recorded in the flowers of $25 \%$ of plant species considered. We are not aware of any other investigation documenting as high a prevalence of nitidulid pollinators at the plant community level. In Müller's (1883) monograph on the floral biology and pollination ecology of central European plants, the only other comparable study known to us, nitidulid beetles were recorded in the flowers of $12 \%$ of 398 species. Estimates of the incidence of nitidulid pollinators in these two thoroughly sampled plant communities are higher than their quantitative representation in recent pollination community studies ("plant-pollinator networks"). Nitidulidae were reported as pollinators for only $3.4 \%$ of the 4056 plant species included in a recent compilation of 295 networks from 123 locations from many places worldwide (Doré et al. 2020), which suggests that the frequency of nitidulid beetles as pollinators is possibly underestimated in recent pollination studies. Given the correlation between nitidulid occurrence and plant phylogeny found in this study, underestimation of nitidulids is expected in small plant species samples that are taxonomically biased (Ollerton et al. 2015) (mean \pm $\mathrm{SE}=25.5 \pm 1.6$ plant species per plant-pollinator network; from data in Doré et al. 2020). 
We found that flower visitation by nitidulid beetles was significantly related to plant phylogeny, their occurrence being clustered on certain plant lineages and remarkably absent from others. Only four plant orders (Asterales, Malvales, Ranunculales, Rosales) out of the 22 orders represented in the sample accounted for $59 \%$ of the plant species in which Nitidulidae appeared in pollinator censuses. Remarkably, the same four orders accounted for $52 \%$ of central European species where Nitidulidae were reported (Müller 1883). In central and mid-western Europe, flowers of Asteraceae, Brassicaceae, Ranunculaceae and Rosaceae are also the most frequent hosts of Brassicogethes aeneus (Ouvrard et al. 2016), the dominant species in our study. These observations suggest geographic consistency in the taxonomic profiles of nitidulid-pollinated flowers, and strengthen the notion of an association between nitidulid pollination and certain plant lineages, independently of habitat or general ecological conditions. These "favoured" plant lineages include the Ranunculales (represented in our sample by species of Ranunculaceae and Berberidaceae), a relatively small group that diverged early, as well as more recent clades such as Malvales, Rosales or Asterales (Smith \& Brown 2018).

As found also in other flower-dwelling beetles, olfactory cues from flowers, particularly from pollen, seem to play an essential role in resource location by Brassicogethes aeneus (Cook et al. 2002; Jürgens \& Dötterl 2004; Jönsson et al. 2007; Corda et al. 2018). Chemical features of pollen, including composition of emitted volatiles and concentration of starch, lipids, proteins, sterols and secondary compounds, vary widely among species, and such variation is closely correlated with phylogeny (Dobson 1988; Dobson et al. 1996; Roulston \& Buchmann 2000; Roulston \& Cane 2000; Roulston et al. 2000; Jürgens \& Dötterl 2004; Zito et al. 2019; Trunz et al. 2020; $\mathrm{Zu}$ et al. 2021). The parsimonious explanation can thus be tentatively advanced that the strong phylogenetic clustering of Nitidulidae pollination in our species sample partly reflects differential foraging responses of nitidulid beetles to phylogenetically-structured interspecific heterogeneity in pollen features which are (or have been) important for location and selection of adult, and also perhaps larval, resources. Since nitidulid beetles sometimes play a dual role as both pollinators and herbivores, phylogenetic conservatism of possible plant defences against them could also have contributed to the strong phylogenetic pattern observed.

Relationships between the frequency of beetle visitation to flowers and ecological (habitat type, altitude; Adedoja et al. 2018, 2020; Herrera 2020) and floral features (size, color; Johnson \& Midgley 2001; Teixido et al. 2011; Streinzer et al. 2019) have been often reported. In the present study, however, none of the environmental (habitat type, elevation) or macroscopic floral features (perianth type and color, flower mass) considered predicted nitidulid visitation rates when plant phylogeny was statistically taken into account. Plant phylogeny was the single most important predictor of the abundance of Nitidulidae pollinators. These results indicate that evolutionarily related plant species were more similar to each other in nitidulid visitation rates than to species more distant phylogenetically, irrespective of their similarities in environmental or gross floral features as perceived by humans. This is illustrated, for instance, by the phylogenetic hotspot of nitidulid incidence associated with Malvales (Fig. 2). Species in this cluster belong to two plant families closely related phylogenetically (Thymelaeaceae and Cistaceae) but with disparate macroscopic floral traits (compare Daphne and Helianthemum in Fig. 1c and $1 \mathrm{j}$ ) and habitat type (shaded forest understory versus sunlit open scrub, respectively). Likewise, the strong association of nitidulid pollinators with flowers of Rosaceae occurred regardless of the broad variety of flower colours, sizes and architectures represented in this family (e.g., Filipendula, Geum, Potentilla, Prunus, Rosa).

\section{CONCLUDING REMARKS}

The present investigation has confirmed for a single beetle lineage the results of Herrera (2020) for the Coleoptera as a whole, namely that at the plant community scale phylogeny was more important than environment or macroscopic floral traits as predictors of the contribution of this group of insects to pollination in the well-preserved montane habitats studied. On one side, our findings indicate that previous results were not an artifact arising from taxonomic and evolutionary heterogeneity of the beetle taxa involved. Before establishing the generality of correlations between 
beetle pollination and plant phylogeny, however, further analyses similar to those conducted here should be performed in other plant communities and for other coleopteran families that are also frequent pollinators (e.g., Dasytidae, Mordellidae, Oedemeridae, Scarabeidae). On the other hand, and perhaps more importantly, this study has provided a case example for the application of an analytical procedure apt to dissect objectively the importance of phylogenetic legacies, gross floral traits and ecological scenarios as predictors of interspecific variation in pollinator composition. Applied to sufficiently large and phylogenetically diverse samples of animal-pollinated plants, the analytical tools used here can help to shed light, from a different angle, on extant controversial issues in pollination research beyond ad hoc interpretations usually applied to small species samples (e.g., the reality of so-called "pollination syndromes"; Herrera 2020, 2021; Krakos \& Austin 2020).

\section{ACKNOWLEDGEMENTS}

Consejería de Medio Ambiente, Junta de Andalucía, granted permission to work in the Sierra de Cazorla and provided invaluable facilities there. Scott Armbruster, Paul Wilson and one anonymous reviewer contributed useful suggestions that improved the manuscript. The research reported in this paper received no specific grant from any funding agency.

\section{APPENDICES}

Additional supporting information may be found in the online version of this article:

AppendixI. Plant species studied, sampling effort, flower visitation by Nitidulid beetles and number of identified individuals per species.

Appendix II. Floral and environmental features of the plant species studied.

\section{REFERENCES}

Adedoja OA, Kehinde T, Samways MJ (2018) Insectflower interaction networks vary among endemic pollinator taxa over an elevation gradient. PLoS ONE 13:e0207453.

Adedoja OA, Kehinde T, Samways MJ (2020) Asynchrony among insect pollinator groups and flowering plants with elevation. Scientific Reports 10:13268.

Alonso C (2004) Early blooming's challenges: extended flowering season, diverse pollinator assemblage and the reproductive success of gynodioecious Daphne laureola. Annals of Botany 93:61-66.

Audisio P (1993) Coleoptera, Nitidulidae-Kateretidae. Fauna d'Italia, 32. Edizioni Calderini, Bologna, Italy.

Bao T, Wang B, Li J, Dilcher D (2019) Pollination of Cretaceous flowers. Proceedings of the National Academy of Sciences USA 116:24707-24711.

Blomberg SP, Garland T (2002) Tempo and mode in evolution: phylogenetic inertia, adaptation and comparative methods. Journal of Evolutionary Biology 15:899-910.

Cai C, Escalona HE, Li L, Yin Z, Huang D, Engel MS (2018) Beetle pollination of cycads in the Mesozoic. Current Biology 28:2806-2812.

Cline AR, Smith TR, Miller K, Moulton M, Whiting M, Audisio P (2014) Molecular phylogeny of Nitidulidae: assessment of subfamilial and tribal classification and formalization of the family Cybocephalidae (Coleoptera: Cucujoidea). Systematic Entomology 39:758-772.

Cook SM, Bartlet E, Murray DA, Williams IH (2002) The role of pollen odour in the attraction of pollen beetles to oilseed rape flowers. Entomologia Experimentalis et Applicata 104:43-50.

Corda SG, Leblanc M, Faure S, Cortesero AM (2018) Impact of flower rewards on phytophagous insects: importance of pollen and nectar for the development of the pollen beetle (Brassicogethes aeneus). ArthropodPlant Interactions 12:779-785.

Crowson RA (1988) Meligethinae as possible pollinators (Coleoptera: Nitidulidae). Entomologia Generalis 14:61-62.

Dobson HEM (1988) Survey of pollen and pollenkitt lipids - chemical cues to flowers visitors? American Journal of Botany 75:170-182.

Dobson HEM, Groth I, Bergström G (1996) Pollen advertisement: chemical contrasts between wholeflower and pollen odors. American Journal of Botany 83:877-885.

Doré M, Fontaine C, Thébault E (2020) Relative effects of anthropogenic pressures, climate, and sampling design on the structure of pollination networks at the global scale. Global Change Biology 27:1266-1280.

Herrera CM (2020) Flower traits, habitat, and phylogeny as predictors of pollinator service: a plant community perspective. Ecological Monographs 90:e01402.

Herrera CM (2021) Unclusterable, underdispersed arrangement of insect-pollinated plants in pollinator niche space. Ecology 102:e03327.

Hsieh TC, Ma KH, Chao A (2016) iNEXT: an R package for rarefaction and extrapolation of species diversity (Hill numbers). Methods in Ecology and Evolution 7:1451-1456. 
Hunt T, Bergsten J, Levkanicova Z, Papadopoulou A, John OS, Wild R, Hammond PM, Ahrens D, Balke M, Caterino MS, et al. (2007) A comprehensive phylogeny of beetles reveals the evolutionary origins of a superradiation. Science 318:1913-1916.

Jin Y, Qian H (2019) V.PhyloMaker: an R package that can generate very large phylogenies for vascular plants. Ecography 42:1353-1359.

Johnson SD, Midgley JJ (2001) Pollination by monkey beetles (Scarabeidae: Hopliini): do color and dark centers of flowers influence alighting behavior? Environmental Entomology 30:861-868.

Jönsson M, Rosdahl K, Anderson P (2007) Responses to olfactory and visual cues by over-wintered and summer generations of the pollen beetle, Meligethes aeneus. Physiological Entomology 32:188-193.

Jürgens A, Dötterl S (2004) Chemical composition of anther volatiles in Ranunculaceae: genera-specific profiles in Anemone, Aquilegia, Caltha, Pulsatilla, Ranunculus, and Trollius species. American Journal of Botany 91:1969-1980.

Jürgens A, Webber AC, Gottsberger G (2000) Floral scent compounds of Amazonian Annonaceae species pollinated by small beetles and thrips. Phytochemistry 55:551-558.

Keck F, Rimet F, Bouchez A, Franc A (2016) phylosignal: an $\mathrm{R}$ package to measure, test, and explore the phylogenetic signal. Ecology and Evolution 6:27742780.

Kono M, Tobe H (2007) Is Cycas revoluta (Cycadaceae) wind- or insect-pollinated? American Journal of Botany 94:847-855.

Krakos KN, Austin MW (2020) Testing pollination syndromes in Oenothera (Onagraceae). Journal of Pollination Ecology 26:52-66.

Losos, JB (2008) Phylogenetic niche conservatism, phylogenetic signal and the relationship between phylogenetic relatedness and ecological similarity among species. Ecology Letters 11:995-1003.

Müller H (1883) The Fertilisation of Flowers. Translated by D. W. Thompson. MacMillan, London, UK.

Münkemüller T, Lavergne S, Bzeznik B, Dray S, Jombart T, Schiffers K, Thuiller W (2012) How to measure and test phylogenetic signal. Methods in Ecology and Evolution 3:743-756.

Nadel H, Peña JE (1994) Identity, behavior, and efficacy of Nitidulid beetles (Coleoptera: Nitidulidae) pollinating commercial Annona species in Florida. Environmental Entomology 23:878-886.

Ollerton J, Rech AR, Waser NM, Price MV (2015) Using the literature to test pollination syndromes - some methodological cautions. Journal of Pollination Ecology 16:119-125.
Ouvrard P, Hicks DM, Mouland M, Nicholls JA, Baldock KCR, Goddard MA, Kunin WE, Potts SG, Thieme T, Veromann E, Stone GN (2016) Molecular taxonomic analysis of the plant associations of adult pollen beetles (Nitidulidae: Meligethinae), and the population structure of Brassicogethes aeneus. Genome 59:11011116.

Paradis E, Schliep K (2018) ape 5.0: an environment for modern phylogenetics and evolutionary analyses in $\mathrm{R}$. Bioinformatics 35:526-528.

Pinheiro J, Bates D, DebRoy S, Sarkar D, R Core Team (2018) nlme: Linear and nonlinear mixed effects models. $\mathrm{R}$ package version 3.1-137, https:/CRAN.Rproject.org/package=nlme.

Podoler H, Galon I, Gazit S (1984) The role of nitidulid beetles in natural pollination of annona in Israel. Acta Oecologica, Oecologia Applicata 5:369-381.

Procheş Ş, Johnson SD (2009) Beetle pollination of the fruit-scented cones of the South African cycad Stangeria eriopus. American Journal of Botany 96:1722-1730.

R Core Team (2020) R: A language and environment for statistical computing. R Foundation for Statistical Computing, Vienna, Austria.

Roulston TH, Buchmann SL (2000) A phylogenetic reconsideration of the pollen starch-pollination correlation. Evolutionary Ecology Research 2:627-643.

Roulston TH, Cane JH (2000) Pollen nutritional content and digestibility for animals. Plant Systematics and Evolution 222:187-209.

Roulston TH, Cane JH, Buchmann SL (2000) What governs protein content of pollen: pollinator preferences, pollen-pistil interactions, or phylogeny? Ecological Monographs 70:617-643.

Schiestl FP, Dötterl S (2012) The evolution of floral scent and olfactory preferences in pollinators: coevolution or pre-existing bias? Evolution 66:2042-2055.

Smith SA, Brown JW (2018) Constructing a broadly inclusive seed plant phylogeny. American Journal of Botany 105:302-314.

Streinzer M, Roth N, Paulus HF, Spaethe J (2019) Color preference and spatial distribution of glaphyrid beetles suggest a key role in the maintenance of the color polymorphism in the peacock anemone (Anemone pavonina, Ranunculaceae) in Northern Greece. Journal of Comparative Physiology A 205:735-743.

Symonds MRE, Blomberg SP (2014) A primer on phylogenetic generalised least squares. Garamszegi LZ (ed) Modern Phylogenetic Comparative Methods and their Application in Evolutionary Biology. Springer, Berlin, pp 105-130.

Teixido AL, Méndez M, Valladares F (2011) Flower size and longevity influence florivory in the large-flowered shrub Cistus ladanifer. Acta Oecologica 37:418-421. 
Trunz V, Lucchetti MA, Benon D, Dorchin A, Desurmont GA, Kast C, Rasmann S, Glauser G, Praz CJ (2020) To bee or not to bee: The 'raison d'être' of toxic secondary compounds in the pollen of Boraginaceae. Functional Ecology 34:1345-1357.

Zito P, Tavella F, Pacifico D, Campanella V, Sajeva M, Carimi F, Ebmer AW, Dötterl S (2019) Interspecific variation of inflorescence scents and insect visitors in
Allium (Amaryllidaceae: Allioideae). Plant Systematics and Evolution 305:727-741.

Zu P, Koch H, Schwery O, Pironon S, Phillips C, Ondo I, Farrell IW, Nes WD, Moore E, Wright GA, Farman DI, Stevenson PC (2021) Pollen sterols are associated with phylogenetics and environment but not with pollinators. New Phytologist 230:1169-1184. 